\title{
Correspondence
}

\section{Comparison of Three-Phase Modulation with Two-Phase and Four-Phase Modulation}

\section{J. R. PIERCE}

\begin{abstract}
For a constant added noise density at high signal-to-noise ratios and for the same error rate, three-phase PSK requires about $0.75 \mathrm{~dB}$ less energy per bit than two-phase or four-phase PSK. However, at very low signal-to-noise ratios, three-phase PSK requires about $0.74 \mathrm{~dB}$ more energy per bit of channel capacity than two-phase or four-phase PSK.
\end{abstract}

Two-phase and four-phase PSK detected with hard limiting and a highly accurate phase reference signal are common modes of information transmission. The purpose of this paper is to contrast, under the above conditions and for a constant spectral density of added Gaussian noise, the performance of three-phase PSK with the (identical) performance of two-phase and four-phase PSK. It is found that for very high signal-tonoise ratios, for the same error rate, three-phase PSK requires about $0.75 \mathrm{~dB}$ less energy per bit than two-phase or fourphase. At very low signal-to-noise ratios, three-phase requires about $0.74 \mathrm{~dB}$ more power per bit of channel capacity than two-phase or four-phase.

No doubt, performances at intermediate signal-to-noise ratios could be determined with the aid of published papers [1]-[4].

In making comparisons, we should note that four-phase PSK can be considered as two independent two-phase channels, detected by means of orthogonal carriers, and each using half of the total signal power. In the comparisons that will be made, the performance of two-phase and four-phase PSK will be equal.

Let us assume a very high signal-to-noise ratio. Then the channel capacity of two-phase PSK is very nearly 1 bit per pulse and the channel capacity of three-phase PSK is very nearly $\log _{2} 3$ bits per pulse. Let our criterion for probability of error be an equal probability of error in sending a long number or word. To transmit the same word by two-phase PSK, we would have to send $\ln 3 / \ln 2$ times as many pulses as in transmitting the word by three-phase PSK. If the pulse-error probability is very small, the word-error probability will be close to the number of pulses times the pulse-error probability. Hence, for equal word-error probabilities, the pulse-error probability in two-phase PSK should be $\ln 2 / \ln 3$ times as great as the pulse error probability in three-phase PSK.

If we are to have equal word-error probabilities at high signal-to-noise ratios, we should have

$$
p_{2}\left(u_{2}\right)=(\ln 3 / \ln 2) p_{3}\left(u_{3}\right)
$$

Paper approved by the Editor for Data Communication Systems of the IEEE Communications Society for publication without oral presentation. Manuscript received December 27, 1978; revised March 3, 1980.

The author is with the Department of Electrical Engineering and the Jet Propulsion Laboratory, California Institute of Technology, Pasadena, CA 91125 .
Here $p_{2}\left(u_{2}\right)$ is the pulse-error probability for two-phase transmission at a signal-to-noise ratio $u_{2}$ and $p_{3}\left(u_{3}\right)$ is the pulse-error probability for a signal-to-noise ratio $u_{3}$.

Equation (2), which is the well-known [5] equation (10.7-13) of [6], gives expressions for $p_{M}\left(u_{M}\right)$ that are valid for $M$ phases and high signal-to-noise ratios $u_{M}$ :

$$
\begin{aligned}
& p_{2}\left(u_{2}\right)=\frac{1 \quad e^{-u_{2}}}{\sqrt{2 \pi} \sqrt{2 u_{2}}} \\
& p_{M}\left(u_{M}\right)=\frac{2}{\sqrt{2 \pi}} \frac{e^{-u_{M} \sin ^{2} \pi / M}}{\sqrt{2 u_{M}} \sin \pi / M}, \quad M \geqslant 3 .
\end{aligned}
$$

At large values of $u_{2}$ and $u_{3}$, the exponential terms in the appropriate expressions dominate, and to satisfy (1) we should have

$$
u_{3}=\frac{4}{3} u_{2}
$$

Thus, for the same noise density, the three-phase bandwidth will be lower by a factor $\ln 2 / \ln 3$ and the three-phase power can be lower by the same factor. Hence, the ratio of power needed for the same error rate for three-phase transmission to that for two-phase transmission is

$$
\frac{4 \ln 2}{3 \ln 3}=0.841 \text { or }-0.75 \mathrm{~dB} \text {. }
$$

This can be generalized for $M$-phase modulation using (2) to

$$
\frac{\ln 2}{\sin ^{2}\left(\frac{\pi}{M}\right) \ln M}
$$

The minimum or best value of this ratio is for $M=3$.

Suppose now that we allow ideal error correction (which achieves operation at channel capacity) and take the ratio of signal power to channel capacity $C$ as our criterion, i.e., energy per bit of channel capacity. This ratio will be lowest for very small signal-to-noise ratios.

From [7, eq. (13)] and a reviewer, we find, using the definition of channel capacity, that for low signal-to-noise ratios, the minimum energy per bit required for $M$-phase modulation is

$$
\begin{aligned}
& P_{2} / C_{2}=(\pi / 2)\left(N_{0} \ln 2\right)=1.09 N_{0} \mathrm{~J} / \mathrm{bit} \\
& P_{M} / C_{M}=\frac{4 \pi}{M^{2} \sin ^{2} \pi / M}\left(N_{0} \ln 2\right) \mathrm{J} / \text { bit, } \quad M \geqslant 3 \\
& \frac{P_{\infty}}{C_{\infty}}=\left(\frac{4}{\pi}\right)\left(N_{0} \ln 2\right)=0.883 N_{0} \mathrm{~J} / \text { bit. }
\end{aligned}
$$


The case $M=\infty$ is that of continuous phase modulation and is obtained as the limit as $M$ becomes large of the expression in (5) for $M \geqslant 3$. We can see that this expression is monotonically decreasing as $M$ increases beyond 3 , so three-phase is indeed the worst case at low signal-to-noise ratios. We find that

$$
\dot{P}_{3} / C_{3}=\left(\frac{16 \pi}{27}\right)\left(N_{0} \ln 2\right)=1.29 N_{0} \mathrm{~J} / \text { bit. }
$$

Thus, for the case of very low signal-to-noise ratios and ideal error correction; the ratio of energy per bit for threephase PSK to energy per bit for two-phase or four-phase PSK is

$$
\frac{32}{27} \text { or }+0.738 \mathrm{~dB} \text {. }
$$

We can also note that the continuous phase case, which corresponds to a channel which passes phase information only but loses all amplitude information, $P_{\infty} / C_{\infty}$ is only

$$
(\pi / 2) /(4 / \pi)=\pi^{2} / 8=1.234=0.91 \mathrm{~dB}
$$

less than the value for phase modulation obtained when $M=2$ or 4. More interesting is the comparison of continuous-phase modulation with the Gaussian channel with no restriction:

$$
\frac{P_{0}}{C}=N_{0} \ln 2=0.693 \mathrm{~J} / \text { bit. }
$$

The continuous-phase channel is worse than this by a factor of

$$
\frac{4}{\pi}=1.273=1.05 \mathrm{~dB} .
$$

We may note that the sum of the losses given in (8) and (9) is

$$
0.91 \mathrm{~dB}+1.05 \mathrm{~dB}=1.96 \mathrm{~dB} .
$$

This is, of course, the familiar $2 / \pi$ loss in the Gaussian channel when we hard limit at a low signal-to-noise ratio.

In summary, for very large signal-to-noise ratios, for the same error rate, three-phase PSK requires about $0.75 \mathrm{~dB}$ less energy per bit than two-phase or four-phase PSK, and the energy per bit at the same error rate is higher for numbers of phases larger than 4 [see (5)]. However, for very low signal-to-noise ratios, the energy per bit of channel capacity is less by about $0.74 \mathrm{~dB}$ for two-phase or four-phase PSK than for three-phase PSK, although a large number of phases is superior to two or four phases.

Thus, it seems that in some sense, three-phase PSK is the best number of phases at very high signal-to-noise ratios and the worst number of phases for very low signal-to-noise ratios.

\section{ACKNÓWLEDGMENT}

The author wishes to thank Dr. E. C. Posner for help beyond the call of duty in rewriting this paper so as to make it acceptable for publication. He also wishes to thank a kind and helpful referee.

\section{REFERENCES}

[1] K. A. Norton, E. L. Shultz, and H. Yarbrough. "The probability of the resultant vector sum of a constant vector plus a Rayleigh distributed vector;" J. Appl. Phys., vol. 23, pp. 137-14 i, Jan. 1952.

[2] C. R. Cahn, " Performance of digital phase-modulation "communication systems," IRE Trans. Commun. Syst., vol. CS-7, pp. 3-6. May 19.59.

[3] A.: D. Wyner, "Bounds on communicátion with polyphase coding," Bell Syst: Tech. J., vol. 45, pp. 523-559. Apr. 1966.

[4] G. Einarsson, "Polyphase coding for a Gaussian channel," Ericcson Tech., no. 2, 1968

[5] E, Arthurs and H. Dym. "On the optimum detection of digital signals in the presence of white Gaussian noise-A geometric interpretation and a study of three basic data transmission systems," IRE Trans. Commun. Syst., vol. CS-10, pp: 336-372 [especially eq. (123)], 1962

[6] J. R. Pierce and E. C. Posner, Introduction to Communication Science and Systems. New. York: Plenum, 1980

[7] W. Doyle and I. S. Reed, "Approximate band-pass limited envelope distributions," IEEE Trans. Inform. Theory, vol. IT-10, pp. $180-185,1964$.

An Inexpensive Automatic Test System for Remote Supervision of Repeatered Lines

\section{K. NAGÁRAJ AND J. K. VERMA}

Abstract-Salient features of a novel automatic fault location scheme for remote supervision of repeatered digital transmission lines is discussed. Looping back repeaters in succession by single command on the power feed line eliminates the use of a separate supervisory cable pair. The scheme has been generalized in such a way as to facilitate its adoption to almost all types of repeaters, thus providing an inexpensive, simple supervisory scheme for low density rural area line transmission hookups.

\section{INTRODUCTION}

The importance of remote fault supervision of repeaters in a digital transmission line having a large number of repeaters is obvious. Various methods of remote fault location on repeatered lines have been in vogue [2]-[4]. Most of these methods need a separate supervisory cable pair and use a special test signal, different from the normal data: As a result, they are expensive and hence suitable only for high-density routes where the fault supervision system can be shared by several repeatered lines.

This paper describes a repeater supervision system which has the following features.

1) It is totally independent of the line code, data rate, and the regeneration method as far as the test sequence is concerned. It is also unaffected by a service channel operating over the phantom pair.

Paper approved by the Editor for Transmission Systems of the IEEE Communications Society for publication without oral presentation. Manuscript received October 22, 1979; revised Janaury 25, 1980 ,

The authors are with the Digital Group, Research Department, Indian Telephone Industries Ltd., Bangalore, India. 